\title{
Closed-Form Solution for the Solow Model with Constant Migration
}

\author{
J.P. JUCHEM NETO ${ }^{*}$, J.C.R. CLAEYSSEN ${ }^{2}$, \\ D. RITELLI ${ }^{3}$ and G. MINGARI SCARPELLO ${ }^{4}$
}

Received on May 17, 2014 / Accepted on March 26, 2015

\begin{abstract}
In this work we deal with the Solow economic growth model, when the labor force is ruled by the Malthusian law added by a constant migration rate. Considering a Cobb-Douglas production function, we prove some stability issues and find a closed-form solution for the emigration case, involving Gauss' Hypergeometric functions. In addition, we prove that, depending on the value of the emigration rate, the economy could collapse, stabilize at a constant level, or grow more slowly than the standard Solow model. Immigration also can be analyzed by the model if the Malthusian manpower is declining.
\end{abstract}

Keywords: Solow growth model, migration, hypergeometric function.

\section{INTRODUCTION}

The Solow one-sector model for economic growth [10], [11] is a landmark in the neoclassic theory of growth, which originated an enormous literature. One of the main assumptions of this model is the labor force ruled by a Malthusian Law, namely an exponential population growth. Joining to this a Cobb-Douglas production function, this model has a well known analytical solution. Relatively recent works have replaced the Malthusian Law by other population growth models. Donghan [5] proposed the replacement by the Verhulst (logistic) Law in the Solow model, without actually solving it, but proving the reaching of a steady state. In the same paper, he proves a comparison, a limit and a stability theorem for the capital per capita evolution, under the assumption of a strictly increasing and bounded labor force. In [9] Mingari Scarpello and Ritelli have shown that for the case of the Verhulst (logistic) Law, the model admits a closedform solution in terms of the Gauss' Hypergeometric function ${ }_{2} F_{1}$. After that, in [4] the Von

\footnotetext{
*Corresponding author: João Plínio Juchem Neto

${ }^{1}$ Centro de Tecnologia de Alegrete, UNIPAMPA - Universidade Federal do Pampa, 97546-550 Alegrete, RS, Brasil. E-mail: plinio@unipampa.edu.br

${ }^{2}$ Instituto de Matemática, UFRGS - Universidade Federal do Rio Grande do Sul, 91509-900 Porto Alegre, RS, Brasil. E-mail: julio@mat.ufrgs.br

${ }^{3}$ Dipartimento di Scienze Statistiche, Università di Bologna, Via Belle Arti 41, Bologna, Italy.

E-mail: daniele.ritelli@unibo.it

${ }^{4}$ Via Negroli 6, Milan, Italy. E-mail: giovannimingari@libero.it
} 
Bertalanffy Law was considered arriving at a closed-form solution involving ${ }_{2} F_{1}$, and in [1] was introduced the Richards Law (a generalized logistic model). In our paper we talk about "migrations" meaning the impact of a constant additional manpower $I$ to the classic Malthusian law which feeds the Solow model. We show that this model also has a closed-form solution in terms of the function ${ }_{2} F_{1}$, for $I<0$ (labor force emigration). This kind of model can be applied to study the brain drain phenomena [7], for example. In the following section we review the classic Solow Model, and in Section 3 we present the model modified by the migration term, discuss the stability and steady-state of the capital and output per capita, and solve it. In Section 4 we discuss briefly the case in which we have immigration $(I>0)$, and in Section 5 we present some simulations. Finally, in Section 6 we state our conclusions.

\section{THE SOLOW MODEL FOR ECONOMIC GROWTH}

The Solow growth model [10] assumes a production function $f$ depending on the accumulated stock of capital $K(t)$ and labor force $L(t)$ at a time $t$, and on a constant factor $A$, a given parameter representing the technological level of the economy:

$$
Y=f(K, L, A), \text { with } K, L, A>0
$$

This production function must meet the following properties:

i) $f(\cdot)$ is an increasing function in both state variables, capital and labor force

$$
\left(\frac{\partial f}{\partial K}>0, \frac{\partial f}{\partial L}>0\right) \text {, with decreasing marginal returns }\left(\frac{\partial^{2} f}{\partial K^{2}}<0, \frac{\partial^{2} f}{\partial L^{2}}<0\right) .
$$

ii) $f(\cdot)$ shall have constant returns to scale, $f(\lambda K, \lambda L)=\lambda f(K, L), \forall \lambda>0$.

iii) $f(\cdot)$ satisfies the Inada conditions:

$$
\lim _{K \rightarrow 0} \frac{\partial f}{\partial K}=\lim _{L \rightarrow 0} \frac{\partial f}{\partial L}=+\infty \text { and } \lim _{K \rightarrow+\infty} \frac{\partial f}{\partial K}=\lim _{L \rightarrow+\infty} \frac{\partial f}{\partial L}=0 .
$$

One of the production function satisfying conditions i, ii and iii, used by Solow in his seminal work was the Cobb-Douglas function giving the output $Y$ as:

$$
Y=A K^{\varphi} L^{1-\varphi}, \text { with } \varphi \in(0,1)
$$

where $\varphi$ closer to 0 means a labor intensive economy, and $\varphi$ closer to 1 a capital intensive one. Considering (2.2), the capital stock dynamics is ruled by the ordinary differential equation:

$$
\dot{K}=s Y-\delta K=s A K^{\varphi} L^{1-\varphi}-\delta K
$$

where $s$ and $\delta$ are the constant saving and depreciation rates, then $s Y$ is the gross investment, and $\delta K$ is the capital depreciation in the whole economy. The labor force dynamics follows the Malthusian Law:

$$
\dot{L}=\alpha L \Rightarrow L(t)=L_{0} e^{\alpha t}
$$


with $L_{0}>0$ being the initial population of workers. Defining the capital per capita:

$$
k(t)=\frac{K(t)}{L(t)}
$$

and the labor force growth rate:

$$
n(t)=\frac{\dot{L(}(t)}{L(t)}
$$

and taking (2.4) into account, we rewrite (2.2) and (2.3) as:

$$
\begin{gathered}
Y=A L(t) k^{\varphi}(t) \\
Y=A L_{0} e^{\alpha t} k^{\varphi}, \text { with } \varphi \in(0,1) \\
\dot{k}+(n(t)+\delta) k=s A k^{\varphi}
\end{gathered}
$$

Then, noting from (2.4) and (2.6) that $n(t)=\alpha$, the solution of the Bernoulli equation (2.9), given the initial capital per capita $k(0)=k_{0}>0$ is:

$$
k(t)=\left[\frac{A s\left(1-e^{-t(1-\varphi)(\alpha+\delta)}\right)+k_{0}^{1-\varphi}(\alpha+\delta) e^{-t(1-\varphi)(\alpha+\delta)}}{\alpha+\delta}\right]^{\frac{1}{1-\varphi}}
$$

and the total output is given plugging (2.10) in (2.8). Observe that the steady-state of the capital per capita, $k_{\infty}$, is given by:

$$
k_{\infty}=\lim _{t \rightarrow+\infty} k(t)=\left(\frac{s A}{\alpha+\delta}\right)^{\frac{1}{1-\varphi}}
$$

Defining the output per capita

$$
y(t)=\frac{Y(t)}{L(t)}=A k^{\varphi}(t)
$$

where we used (2.7), such output, in the long run, will tend to:

$$
y_{\infty}=\lim _{t \rightarrow+\infty} A k^{\varphi}(t)=A\left(\frac{s A}{\alpha+\delta}\right)^{\frac{\varphi}{1-\varphi}} .
$$

\section{THE SOLOW GROWTH MODEL WITH MIGRATION}

In this work we will add a constant migration rate $I$ in the r.h.s. of the differential equation (2.4) that governs the growth of the labor force:

$$
\dot{L}=\alpha L+I
$$

whose solution is:

$$
L(t)=-\frac{I}{\alpha}+\left(L_{0}+\frac{I}{\alpha}\right) e^{\alpha t}
$$


Observe that, making $I=0$, we recover the exponential law (2.4), and besides of that $\lim _{t \rightarrow+\infty} \bar{n}(t)=\alpha$ (the over score will mark hereinafter all the ratios relevant to migration model) where, in this case:

$$
\bar{n}(t)=\frac{\dot{L}(t)}{L(t)}=\frac{\alpha\left(\alpha L_{0}+I\right)}{\alpha L_{0}+I\left(1-e^{-\alpha t}\right)}
$$

That is, in the long run, the variation rate of the labor force tends to the same value $\alpha$ of the exponential law. In principle, the migration rate I (number of individuals per time) is an exogenous variable that could be positive (immigration, workers entering the labor force at a constant rate), negative (emigration, workers leaving the labor force) or zero (no migration). Then, considering (3.3) and the change of variable

$$
z=\bar{k}^{1-\varphi}
$$

we can rewrite (2.9) as the following linear differential equation, now in $z(t)$ :

$$
\dot{z}-(\varphi-1)(\delta+\bar{n}(t)) z=(1-\varphi) s A
$$

subject to the initial condition $z(0)=z_{0}=k_{0}^{1-\varphi}$. Defining the integrating factor:

$$
H(t)=(\varphi-1) \int_{0}^{t}(\delta+\bar{n}(\tau)) d \tau=(\varphi-1) \delta t+(\varphi-1) \ln \left(\frac{L(t)}{L_{0}}\right)
$$

then the solution of (3.5) is given by:

$$
z(t)=z_{0} e^{H(t)}+(1-\varphi) s A t e^{H(t)} \int_{0}^{t} e^{-H(\tau)} d \tau .
$$

\subsection{Stability and Steady-State}

Before starting to formulate an analytical expression for (3.7), and therefore for $\bar{k}(t)$, let us discuss the stability and asymptotes of $\bar{k}(t)$, considering $\alpha, L_{0}>0$.

\section{Proposition 1.}

i) The capital per capita $\bar{k}(t)$ is globally asymptotically stable for $I \in\left[-\alpha L_{0}, 0\right]$.

ii) The capital per capita goes to infinity within a finite time if $I \in\left(-\infty,-\alpha L_{0}\right)$, that is, $\lim _{t \rightarrow t^{*}} \bar{k}(t)=+\infty$ where $t^{*}=\frac{1}{\alpha} \ln \left(\frac{I}{\alpha L_{0}+I}\right)$.

\section{Proof.}

i) If all the trajectories which start sufficiently close to a solution under test, not only stay close, but also eventually approach it as $t \rightarrow+\infty$, then such a solution is named globally asymptotically stable. We will show that $z(t)$ is globally asymptotically stable in the interval $I \in\left[-\alpha L_{0}, 0\right]$. Then, by the continuity of the change of variable (3.4), we conclude 
that $\bar{k}(t)$ is also globally asymptotically stable. First, let us consider a solution $v(t)$ of (3.5) subjected to the arbitrary positive initial condition $v(0)=v_{0}>0$. This solution is given by:

$$
v(t)=v_{0} e^{H(t)}+(1-\varphi) s A t e^{H(t)} \int_{0}^{t} e^{-H(\tau)} d \tau .
$$

Considering (3.7) and (3.8), we have that $|z(t)-v(t)|=\left|z_{0}-v_{0}\right| e^{H(t)}$. By (3.2), and for $I \in\left[-\alpha L_{0}, 0\right]$, we have that $L(t) \rightarrow+\infty$ as $t \rightarrow+\infty$. Besides of that, because $\varphi \in(0,1), \lim _{t \rightarrow+\infty} H(t)=-\infty$ by (3.6). Therefore: $\lim _{t \rightarrow+\infty}|z(t)-v(t)|=0$ and then, we infer the global asymptotic stability of $z(t)$ and $\bar{k}(t)$.

ii) If $I \in\left(-\infty,-\alpha L_{0}\right)$, we have that $L\left(t^{*}\right)=0$ for a finite time $t^{*}$ given by

$$
t^{*}=\frac{1}{\alpha} \ln \left(\frac{I}{\alpha L_{0}+I}\right) .
$$

This implies that $\lim _{t \rightarrow t^{*}} H(t)=+\infty$, and by (3.7), that $\lim _{t \rightarrow t^{*}} z(t)=+\infty$. Then, $t=t^{*}$ is a vertical asymptote for both $z(t)$ and $\bar{k}(t)$.

Proposition 2. The steady-state capital per capita $\bar{k}_{\infty}$ is given by:
i) $\bar{k}_{\infty}=\left(\frac{s A}{\delta+\alpha}\right)^{\frac{1}{1-\varphi}}$, if $I \in\left(-\alpha L_{0}, 0\right]$.
ii) $\bar{k}_{\infty}=\left(\frac{s A}{\delta}\right)^{\frac{1}{1-\varphi}}$, if $I=-\alpha L_{0}$.

Proof. First, observe that we can find the horizontal asymptote $z_{\infty}$ for $z(t)$ making $\dot{z}=$ 0 in the differential equation (3.5), isolating $z(t)$, and taking its limit as $t \rightarrow+\infty, z_{\infty}=$ $\lim _{t \rightarrow+\infty} \frac{s A}{\delta+\bar{n}(t)}$, where $\bar{n}(t)$ is given by (3.3). Therefore, by (3.4), $\bar{k}_{\infty}$ can be written as:

$$
\bar{k}_{\infty}=\left(\frac{s A}{\delta+\lim _{t \rightarrow+\infty} \bar{n}(t)}\right)^{\frac{1}{1-\varphi}} .
$$

i) For $I \in\left(-\alpha L_{0}, 0\right]$, we have that $\lim _{t \rightarrow+\infty} \bar{n}(t)=\alpha$ by (3.3), and then $\bar{k}_{\infty}=\left(\frac{s A}{\delta+\alpha}\right)^{\frac{1}{1-\varphi}}$.

ii) If $I=-\alpha L_{0}$, the labor force remains constant over time, and again by $(3.3), \bar{n}(t) \equiv 0$. Therefore $\bar{k}_{\infty}=\left(\frac{s A}{\delta}\right)^{\frac{1}{1-\varphi}}$.

Using (2.12), and the above results, we get the propositions below, involving the output per capita $\bar{y}(t)$.

\section{Proposition 3.}

i) The output per capita $\bar{y}(t)$ is globally asymptotically stable for $I \in\left[-\alpha L_{0}, 0\right]$. 
ii) The output per capita goes to infinity at a finite time if $I \in\left(-\infty,-\alpha L_{0}\right)$, that is, $\lim _{t \rightarrow t^{*}} \bar{y}(t)=+\infty$ where $t^{*}=\frac{1}{\alpha} \ln \left(\frac{I}{\alpha L_{0}+I}\right)$.

Proposition 4. The steady-state output per capita $\bar{y}_{\infty}$ is given by:
i) $\bar{y}_{\infty}=A\left(\frac{s A}{\delta+\alpha}\right)^{\frac{\varphi}{1-\varphi}}$, if $I \in\left(-\alpha L_{0}, 0\right]$.
ii) $\bar{y}_{\infty}=A\left(\frac{s A}{\delta}\right)^{\frac{\varphi}{1-\varphi}}$, if $I=-\alpha L_{0}$.

Note that the labor force emigration critical value capable of offsetting the population growth is $I=-\alpha L_{0}$, maintaining the labor force constant. In this case, by Propositions 2(ii) and 4(ii), we have that the level of capital and output per capita in the long-run are greater than in the case without emigration (2.11) and (2.13):

$$
\begin{aligned}
& \bar{k}_{\infty}=\left(\frac{s A}{\delta}\right)^{\frac{1}{1-\varphi}}>\left(\frac{s A}{\delta+\alpha}\right)^{\frac{1}{1-\varphi}}=k_{\infty} \\
& \bar{y}_{\infty}=A\left(\frac{s A}{\delta}\right)^{\frac{\varphi}{1-\varphi}}>A\left(\frac{s A}{\delta+\alpha}\right)^{\frac{\varphi}{1-\varphi}}=y_{\infty}
\end{aligned}
$$

because $\alpha>0$. Otherwise, if $I \in\left(-\alpha L_{0}, 0\right]$, we can see, by Propositions 2(i) and 4( $\left.i\right)$, that the equality holds, i.e.:

$$
\bar{k}_{\infty}=k_{\infty} \text { and } \bar{y}_{\infty}=y_{\infty}
$$

Then it is clear the criticality of the emigration threshold $I=-\alpha L_{0}$ : below it both $\bar{k}_{\infty}$ and $\bar{y}_{\infty}$ are greater than the correspondent classic values, while above it, they equate the classic ones.

\subsection{Closed-form Solution for $I<0$}

Integrating (3.7) and coming back to $\bar{k}(t)$, we find that:

$$
\bar{k}(t)=\left[e^{H(t)}\left(\bar{k}_{0}^{1-\varphi}+(1-\varphi) s A \int_{0}^{t} e^{-H(\tau)} d \tau\right)\right]^{\frac{1}{1-\varphi}}
$$

where by (3.6):

$$
H(t)=(\varphi-1) \int_{0}^{t}(\delta+\bar{n}(\tau)) d \tau=(\varphi-1) \delta t+\ln \left[e^{\alpha t}\left(1+\frac{I}{\alpha L_{0}}\right)-\frac{I}{\alpha L_{0}}\right]^{\varphi-1}
$$

The integral in (3.12) is given by:

$$
\mathfrak{\Im}=\int_{0}^{t} e^{-H(\tau)} d \tau=\int_{0}^{t} e^{(1-\varphi) \delta \tau}\left[e^{\alpha \tau}\left(1+\frac{I}{\alpha L_{0}}\right)-\frac{I}{\alpha L_{0}}\right]^{1-\varphi} d \tau
$$

Following [9] we carry out the change of variable $u=e^{\alpha \tau}$ :

$$
\mathfrak{\Im}=\frac{1}{\alpha} \int_{1}^{e^{\alpha t}} u^{\frac{(1-\varphi) \delta}{\alpha}-1}\left[-\frac{I}{\alpha L_{0}}+\left(1+\frac{I}{\alpha L_{0}}\right) u\right]^{1-\varphi} d u
$$




$$
=\frac{1}{\alpha}\left(-\frac{I}{\alpha L_{0}}\right)^{1-\varphi} \int_{1}^{e^{\alpha t}} u^{\frac{(1-\varphi) \delta}{\alpha}-1}\left[1-\left(1+\frac{\alpha L_{0}}{I}\right) u\right]^{1-\varphi} d u
$$

where in the last step we have to consider $I<0$, in order to guarantee a real value to the expression $\left(-\frac{I}{\alpha L_{0}}\right)^{1-\varphi}$.

The last integral relates to the Euler's integral representation of the Gauss' Hypergeometric Function ${ }_{2} F_{1}$ (see [2], [6], [8]):

$$
\begin{aligned}
{ }_{2} F_{1}\left(\begin{array}{c}
a, b \\
c
\end{array} \mid x\right) & =\sum_{n=0}^{\infty} \frac{(a)_{n}(b)_{n}}{(c)_{n}} \frac{z^{n}}{n !} \\
& =\frac{\Gamma(c)}{\Gamma(c-b) \Gamma(b)} \int_{0}^{1} t^{b-1}(1-t)^{c-b-1}(1-z t)^{-a} d t
\end{aligned}
$$

where $(.)_{n}$ is a Pochhammer symbol. The series is convergent for any $a, b, c$ if $|z|<1$, and for $\operatorname{Re}\{a+b-c\}<0$ if $|z|=1$. For the integral representation is required $\operatorname{Re}(c)>\operatorname{Re}(b)>0$. Here $\Gamma(z)$ denotes the Gamma Function. A quick overview of Gauss' Hypergeometric Function can be found in [3].

After some algebra, we can write the integral $\mathfrak{s}$ in the closed-form

$$
\mathfrak{\Im}=\frac{1}{\alpha}\left(-\frac{I}{\alpha L_{0}}\right)^{1-\varphi}\left(\Im_{t}-\Im_{0}\right)
$$

where:

$$
\begin{aligned}
& \Im_{0}=\frac{\alpha}{(1-\varphi) \delta}{ }_{2} F_{1}\left(\begin{array}{c|c}
a, b & z_{1} \\
c &
\end{array}\right) \\
& \Im_{t}=\frac{\alpha e^{(1-\varphi) \delta t}}{(1-\varphi) \delta}{ }_{2} F_{1}\left(\begin{array}{c|c}
a, b & z_{2} \\
c &
\end{array}\right)
\end{aligned}
$$

and $a, b, c, z_{1}, z_{2}$ are defined as:

$$
\begin{aligned}
& a=\varphi-1, \quad b=\frac{(1-\varphi) \delta}{\alpha}, \quad c=\frac{(1-\varphi) \delta}{\alpha}+1 \\
& z_{1}=1+\frac{\alpha L_{0}}{I}, \quad z_{2}=\left(1+\frac{\alpha L_{0}}{I}\right) e^{\alpha t}
\end{aligned}
$$

Finally, the capital per capita (3.12) is given by:

$$
\begin{gathered}
\bar{k}(t)=\left\{e^{(\varphi-1) \delta t}\left[e^{\alpha t}\left(1+\frac{I}{\alpha L_{0}}\right)-\frac{I}{\alpha L_{0}}\right]^{\varphi-1}\right. \\
\left.\times\left[\bar{k}_{0}^{1-\varphi}+\frac{s(1-\varphi) A}{\alpha}\left(-\frac{I}{\alpha L_{0}}\right)^{1-\varphi}\left(\mathfrak{\Im}_{t}-\mathfrak{\Im}_{0}\right)\right]\right\}^{\frac{1}{1-\varphi}}
\end{gathered}
$$


where $I<0, \varphi \in(0,1), L_{0}, \alpha, s, \delta>0$ and $\mathfrak{s}_{0}, \mathfrak{\Im}_{t}$ are given by (3.14)-(3.16). Plugging (3.2) and (3.17) in (2.7) we get the gross output of the economy:

$$
\bar{Y}(t)=\frac{A}{\alpha}\left[\left(\alpha L_{0}+I\right) e^{\alpha t}-I\right] \bar{k}(t)^{\varphi}
$$

which describes the total production of the economy when the natural Malthusian labor force growth is modified by a constant emigration rate of workers. This framework could be used in the analysis of brain drain phenomena, for instance. Observe that if the emigration rate is too strong $\left(I<-\alpha L_{0}\right)$, the economy collapses at a finite time; if the emigration is equal to minus the initial labor force rate, the total output converges to $-\frac{I A}{\alpha}\left(\frac{s A}{\delta}\right)^{\frac{\varphi}{1-\varphi}}$; and if $-\alpha L_{0}<I<0$ we have an output always minor than in the absence of emigration. As we will see in the next section, (3.18) is also valid in a scenario where there is immigration, but in a declining labor force environmental, that nowadays is a realistic one in some developed countries.

\section{WHAT ABOUT IMMIGRATION?}

Due to the structure of our described growth model, a transient including immigration cannot be treated: in fact with $I>0$ and $\alpha>0$, (3.1) gives an exponential manpower, $L(t)=$ $\frac{1}{\alpha}\left[\left(\alpha L_{0}+I\right) e^{\alpha t}-I\right]$, growing during time well faster than the Malthusian $L_{0} e^{\alpha t}$. In such a way there would exist a finite instant defined by $t^{*}=\frac{1}{\alpha} \ln \left(\frac{I}{\alpha L_{0}+I}\right)$ at which by (3.3) the coefficient $\bar{n}(t)$ in (3.5) becomes infinite, so that all the microeconomic model would burst out. Thus for a positive trend $\alpha>0$ no situation $I>0$ is possible, unless $t<t^{*}$.

This can be seen also through (3.17) in which the reality of $\left(\frac{-I}{\alpha L_{0}}\right)^{1-\varphi}$ requires $\alpha$ and $I$ to be opposite in sign. Then an immigration $(I>0)$ could be in principle "balanced" by a negative trend $(\alpha<0)$ of the Malthus manpower law. In this case the pole $t^{*}$ will be negative for an initial population such that the addition of the immigration rate to the initial labor force rate is positive. Otherwise the pole of $\bar{n}(t)$ would be innoxius and not real.

The sense of all this is that an exogenous contribution of manpower can be absorbed by the system whenever its labor force is declining. Otherwise in presence of a (also slightly) own growing labor force, whichever immigration will push the system sooner or later beyond its capabilities and then will cause its explosion.

It is straightforward to show that if $\alpha<0$ and $I>0$, then the capital and output per capita steady-states are given by

$$
\begin{gathered}
\bar{k}_{\infty}=\left(\frac{s A}{\delta}\right)^{\frac{1}{1-\varphi}}<\left(\frac{s A}{\delta+\alpha}\right)^{\frac{1}{1-\varphi}}=k_{\infty} \\
\bar{y}_{\infty}=A\left(\frac{s A}{\delta}\right)^{\frac{\varphi}{1-\varphi}}<A\left(\frac{s A}{\delta+\alpha}\right)^{\frac{1}{1-\varphi}}=k_{\infty}
\end{gathered}
$$

where we compared with the steady-states of the Malthusian Law (2.11) and (2.13). 
As a last remark, observe that in the case of a decaying manpower Malthusian Law, the total output steaty-state $Y_{\infty}$ is zero. But when we balance it with a positive migration rate $(I>0)$, we have that:

$$
\bar{Y}_{\infty}=-\frac{I A}{\alpha}\left(\frac{s A}{\delta}\right)^{\frac{\varphi}{1-\varphi}}>0=Y_{\infty}
$$

\section{SAMPLE PROBLEMS}

Considering the set of parameters $\alpha=0.02, \varphi=0.5, \delta=0.05, s=0.06, A=1, \bar{k}_{0}=$ 200, $L_{0}=100$, we plotted the gross output of the economy given by (3.18) in Figure 1 . Note that: when $I=-\alpha L_{0}=-2$, the labor force emigration compensates the population growth, implying a constant labor force, and the convergence of the total output $\bar{Y}(t)$ of the economy to a constant value in the long run, as $t \rightarrow+\infty$; when $I \in\left(-\alpha L_{0}, 0\right)=(-2,0)$, as a result of emigration, the labor force grows more slowly than in the classic Solow model, which causes a slow increase in the total output $\bar{Y}(t)$; if $I=0$, we have no emigration, and $\bar{Y}(t)$ grows in an exponential way, given by classic Solow model; if $I \in\left(-\infty,-\alpha L_{0}\right)=(-\infty,-2)$, the emigration rate is greater than the natural growth rate of the labor force, causing its extinction. In this case, $\bar{Y}(t)$ becomes zero at a finite time $t^{*}$, leading the economy to a collapse.

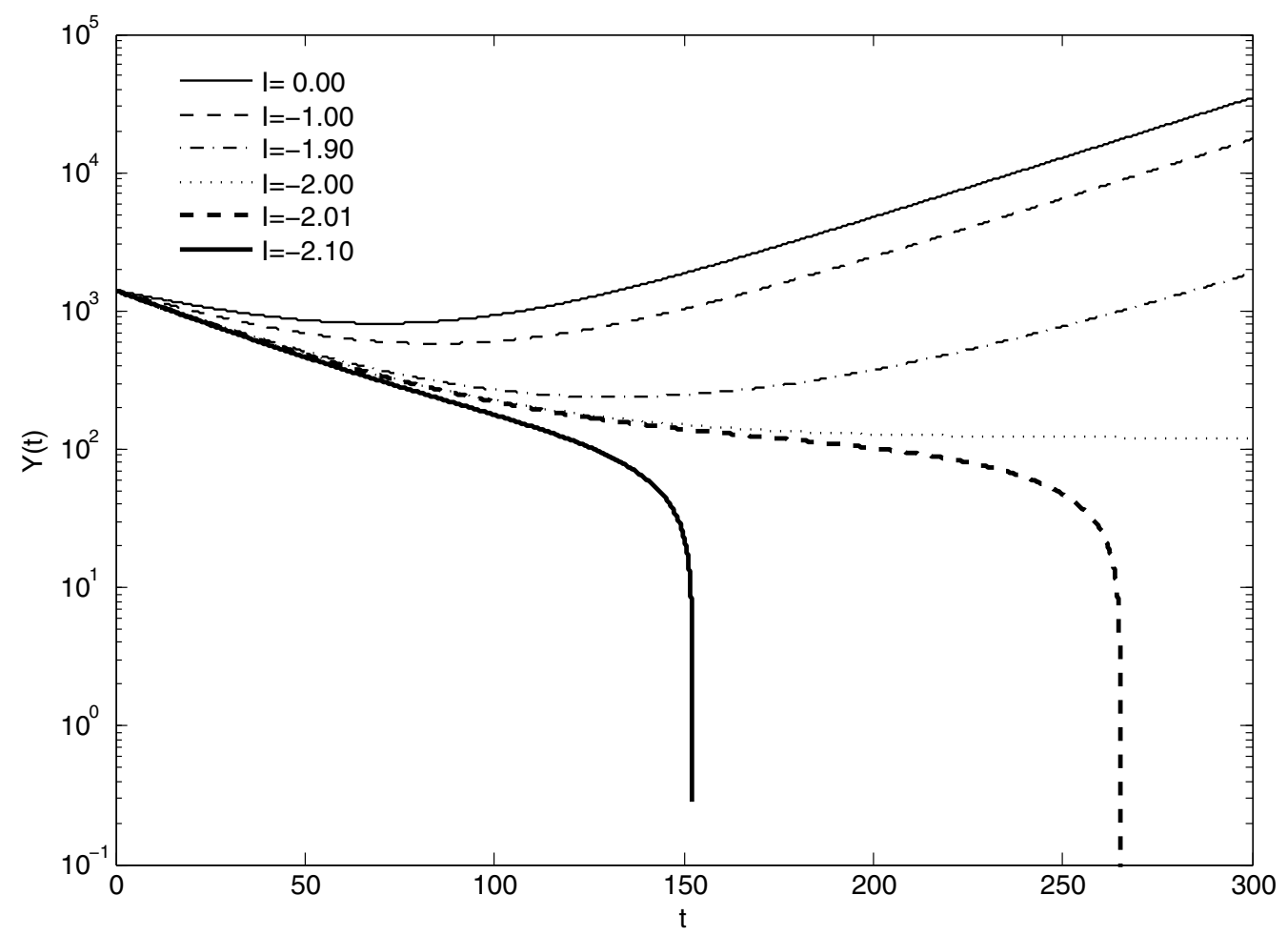

Figure 1: Gross Output versus Time $(I<0, \alpha>0)$. 
Observe that in this last case, from the proof of Proposition 1(i), the time $t^{*}$ at which the economy collapses is given by:

$$
t^{*}=\frac{1}{\alpha} \ln \left(\frac{I}{\alpha L_{0}+I}\right)
$$

when $L\left(t^{*}\right)=0$, and then $Y\left(t^{*}\right)=0$, from (3.18).

In Figure 2 we show the capital per capita evolution, for some values of $I$ : when $I=-\alpha L_{0}=$ -2 , the capital per capita get steady at an upper level than the Solow model, when $I=0$ (see result (3.10)); when $I=-1 \in\left(-\alpha L_{0}, 0\right)=(-2,0), \bar{k}(t)$ in the short term are greater when there is emigration, than when there is not, but when $t \rightarrow+\infty$, it tends to the same level given by the Solow model (see result (3.11)); if $I \rightarrow 0^{-}$, we recover the behaviour of the classic Solow model; if $I=-2.1 \in\left(-\infty,-\alpha L_{0}\right)=(-\infty,-2), L(t)=0$ in a finite time $t^{*}$, which implies that $\bar{k}(t) \rightarrow+\infty$ as $t \rightarrow t^{*}$, but remember that in this case we have the collapse of the economy, with $\bar{Y}\left(t^{*}\right)=0$, as noted in Figure 1 (see Proposition 1(ii)).

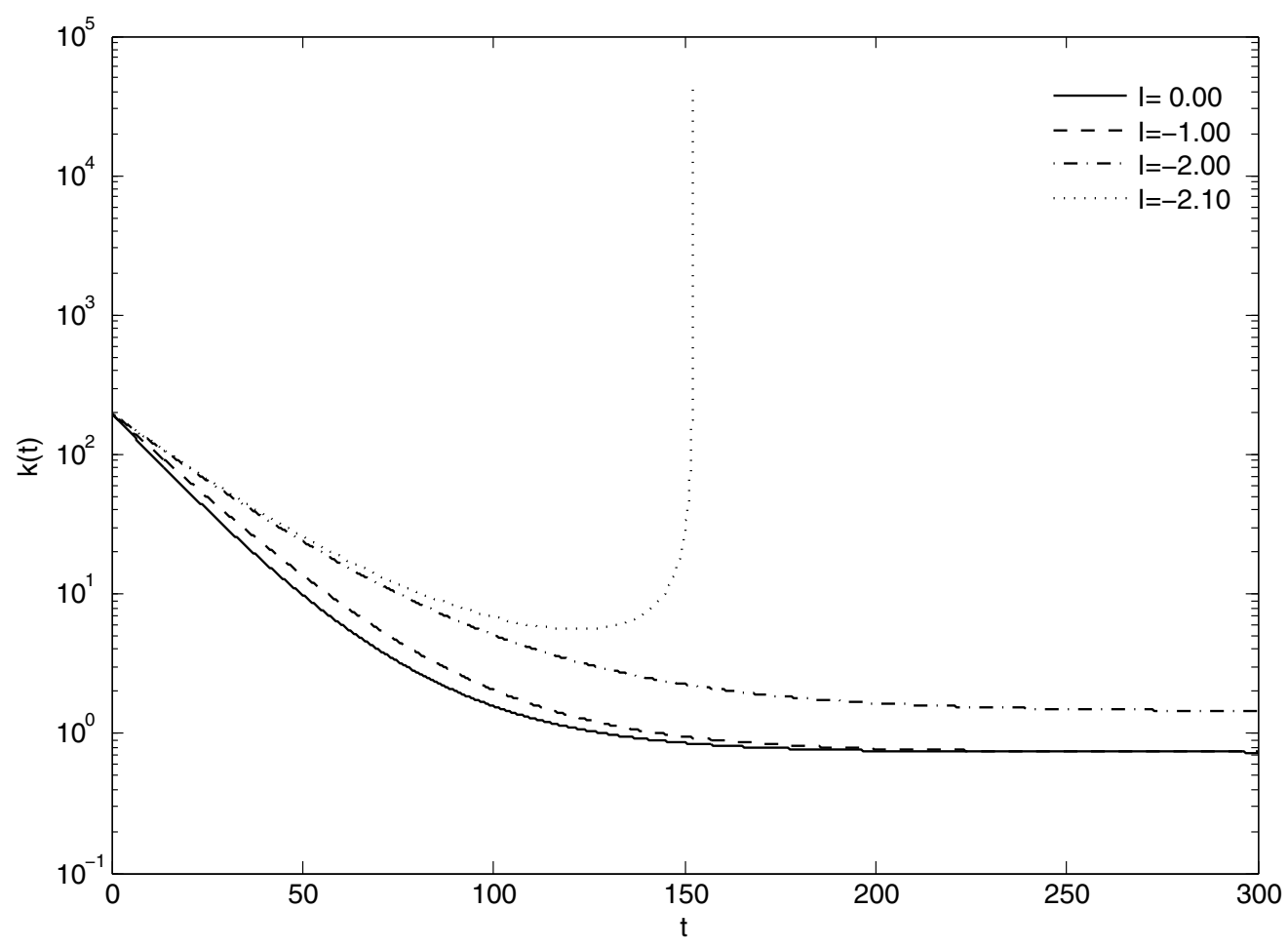

Figure 2: Capital per Capita versus Time $(I<0, \alpha>0)$.

In Figure 3 we can verify that the solution of our model convergences to the classic Solow model as $I \rightarrow 0^{-}$. In a scenario with decreasing labor force and immigration, we verify relations (4.3) and (4.1) in Figures 4 and 5, respectively, for $\alpha=-0.02$ and the other parameters equal to those of the above simulations. 


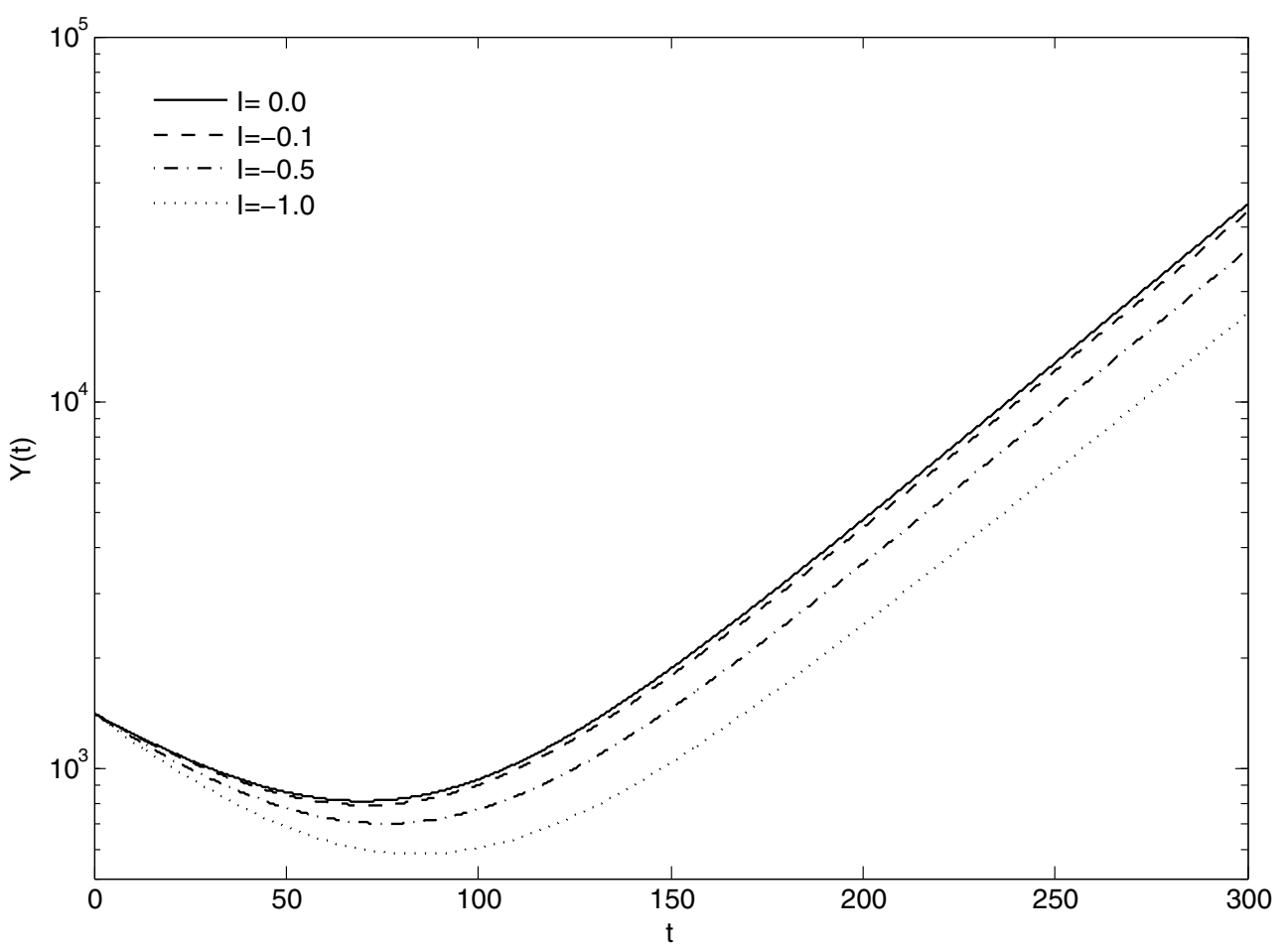

Figure 3: Gross output versus time and its convergence to the classic model as $I \rightarrow 0^{-}$ $(I<0, \alpha>0)$.

\section{CONCLUSIONS}

In this paper we derived a closed-form solution for the Solow growth model involving the Gauss' Hypergeometric Function ${ }_{2} F_{1}$, assuming the dynamics of the labor force to follow a Malthusian Law with a constant emigration rate $I<0$, such that when $I \rightarrow 0^{-}$the classic Solow model is recovered. We also proved the global asymptotic stability of the capital and output per capita for $I \in\left[-\alpha L_{0}, 0\right]$, although the closed-form solution presented is only valid in the interval $I \in\left[-\alpha L_{0}, 0\right)$. Besides of that, our analytical simulations for a specified set of parameters prove that the range of emigration $I \in\left(-\infty,-\alpha L_{0}\right)$ is such that the labor force becomes zero at a finite time, leading the economy to a collapse. For $I=-\alpha L_{0}$ the labor force keeps constant, which implies that the total output tends to a constant limit greater than zero, i.e., the economy stagnates in the long run, and both capital and production per capita are over the time always greater than the classic case, and in the long run tend to a stationary level also greater than the classic one. For $I \in\left(-\alpha L_{0}, 0\right)$, we have a level of emigration capable of inducing labor force increases more slowly than the classic case, implying: greater levels in capital and production per capita in the short run, but tending to the same level of the classic Solow model as $t \rightarrow \infty$. Finally, immigration $(I>0)$ can be included in our transient model if and only if the Malthusian manpower goes down on its own $(\alpha<0)$. 


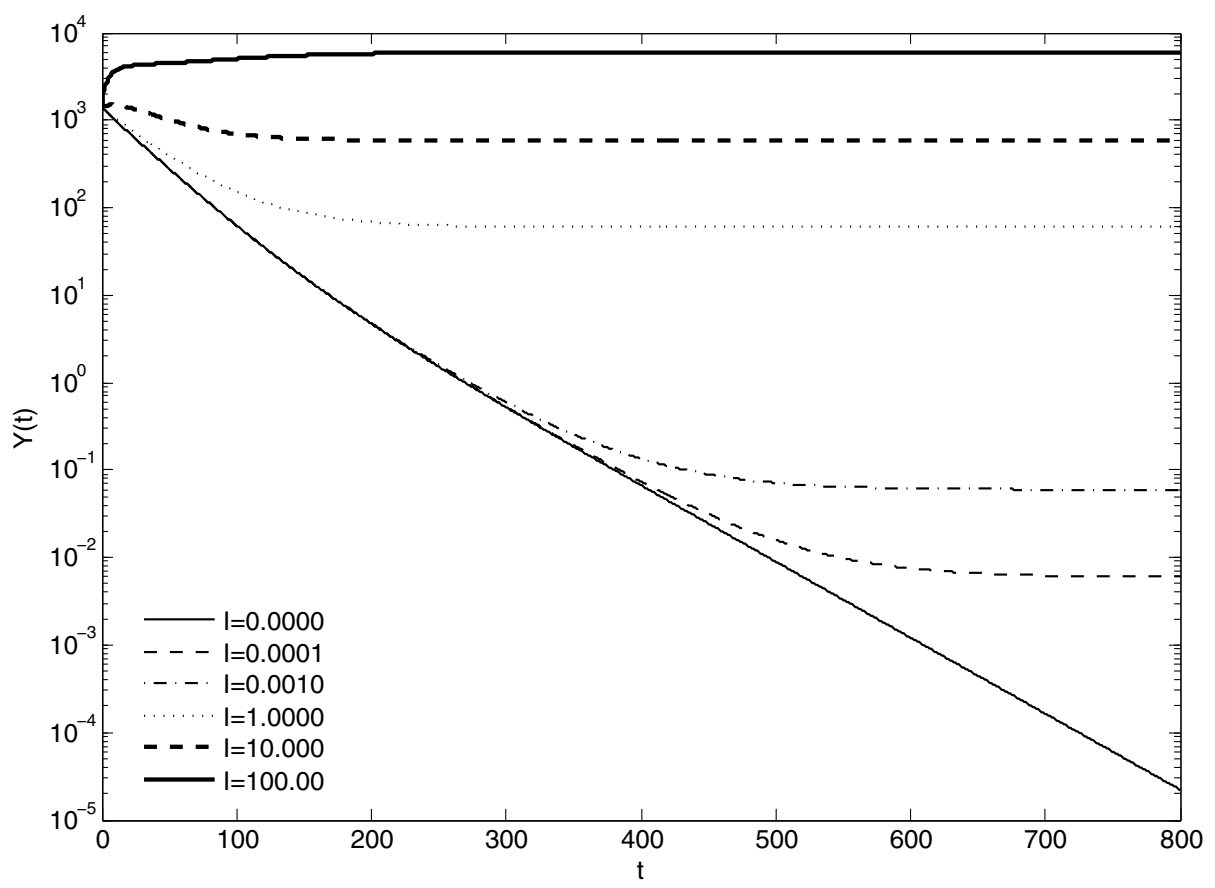

Figure 4: Gross Output versus Time $(I>0, \alpha<0)$.

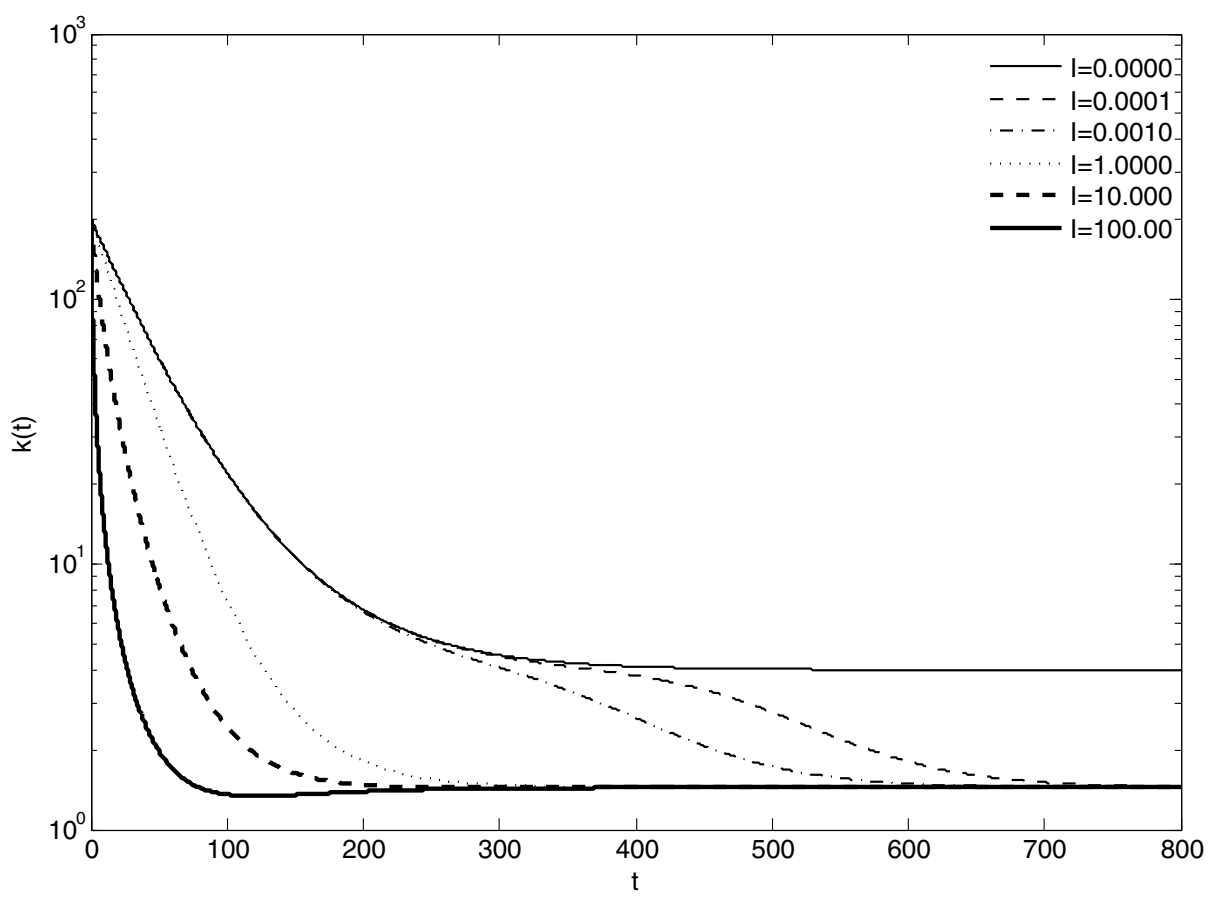

Figure 5: Capital per Capita versus Time $(I>0, \alpha<0)$. 
RESUMO. Neste trabalho consideramos o modelo de crescimento econômico de Solow, quando a força de trabalho é governada pela lei de Malthus adicionada por uma taxa de migração constante. Considerando a função de produção de Cobb-Douglas, provamos alguns resultados de estabilidade e encontramos uma solução em forma fechada, envolvendo funções hipergeométricas de Gauss, para o caso em que há emigração. Além disso, provamos que, dependendo do valor da taxa de emigração, a economia pode entrar em colapso, estabilizar em um nível constante, ou crescer mais vagarosamente do que o modelo de Solow padrão. O caso em que há imigração também pode ser analisado pelo modelo, desde que a taxa de crescimento orgânico de trabalhadores na Lei de Malthus seja negativa.

Palavras-chave: Modelo de crescimento de Solow, migração, função hipergeométrica.

\section{REFERENCES}

[1] E. Accinelli \& J.G. Brida. Re-formulation of the Solow economic growth model whit the Richards population growth law. GE, Growth, Math methods 0508006, EconWPA, 2005. Available in: http://ideas.repec.org/p/wpa/wuwpge/0508006.html.

[2] G.E. Andrews, R. Askey \& R. Roy. Special Functions, Cambrigde Press (1999).

[3] R. Boucekkine \& J.R. Ruiz-Tamarit. Special functions for the study of economic dynamics: The case of the Lucas-Uzawa model. Journal of Mathematical Economics, 44 (2008), 33-54.

[4] J.G. Brida \& E.J.L. Maldonado. Closed form solutions to a generalization of the Solow growth model. Applied Mathematical Sciences, 1(40) (2007), 1991-2000.

[5] C. Donghan. An Improved Solow Model. Chinese Quarterly Journal of Mathematics, 13(2) (1998), 72-78.

[6] A. Erdélyi (Editor). Higher Transcendental Functions - Volume I, McGraw-Hill Book Company, USA, (1953).

[7] P. Pieretti \& B. Zou. Brain drain and factor complementarity. Economic Modelling (2008), doi: 10.1016/j.econmod.2008.08.002.

[8] E.D. Rainville. Special Functions. The Macmillian Company, New York (1960).

[9] G. Mingari Scarpello \& D. Ritelli. The Solow Model Improved Through the Logistic Manpower Growth Law. Annali Università di Ferrara - Sez VII - Sc. Mat., II (2003), 73-83.

[10] R. Solow. A contribution to the theory of economic growth. Quarterly Journal of Economics, LXX (1956), 65-94, February.

[11] T.W. Swan. Economic Growth and Capital Accumulation. Economic Record, 32 (1956), 334-361. 